\title{
Method for improving the precision of measurement for the condition of wear surfaces on Shinkansen trains
}

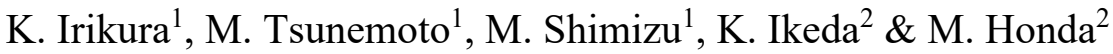 \\ ${ }^{I}$ Power Supply Technology Division, \\ Railway Technical Research Institute, Japan \\ ${ }^{2}$ East Japan Railway Company, Japan
}

\begin{abstract}
An electric inspection car measures the wear area of the contact wire by laser to calculate the residual diameter of the contact wire. The contact wire in a Shinkansen tunnel rarely has any gloss along the wear surface. In that case, the measured wear area has some error. The authors considered the wear condition difference between the contact wire with gloss and that without gloss by measuring the two-dimensional cross-sectional shapes and the image of the wear surface. Keywords: contact wire, two-dimensional cross-section, image of wear surface, wear, tunnel, gloss.
\end{abstract}

\section{Introduction}

Regular maintenance of the contact wire is essential because of gradual wear due to sliding of the pantograph. The basic technique of the maintenance of contact wire is nothing; the question is how to manage its residual diameter. In general, the residual diameter is calculated based on the measured width of the wear surface by the electric inspection car in the Shinkansen system [1,2].

In recent years, some gloss has been seen at the edge of the wear surface where there is an increase in speed in some sections of the Shinkansen [3].

Because of the diffused reflection of the laser beam that is estimated to have occurred by the influence of the gloss, some cases of errors of the residual diameter measurements by the electrical inspection car are reported [4]. 
This paper reports the results of the study on processing the two-dimensional cross-sectional shape data and the wear surface image by computer to solve this problem.

\section{Measurement}

\subsection{Summary}

In order to compare the accurate residual diameter and the measured value by the inspection car, the authors conducted a recording of the measured values by the electrical inspection car and the static measurement of the residual diameter on the maintenance car at low speed (about $4 \mathrm{~km} / \mathrm{h}$ ). Fig. 1 shows the state of the static measurement of the residual diameter. Table 1 shows the measuring instruments and measurement accuracies of the static measurement of the residual diameter and the electrical inspection car. In the static measurement, the twodimensional laser displacement sensor, line camera, and lighting were additionally prepared for the method of measuring residual diameters proposed in this paper.

Four sections were selected for the measurement with consideration on factors such as the presence or absence of tunnel and overhead contact lines structure and the kinds of contact wires. Table 2 shows the measured contact wire sections.

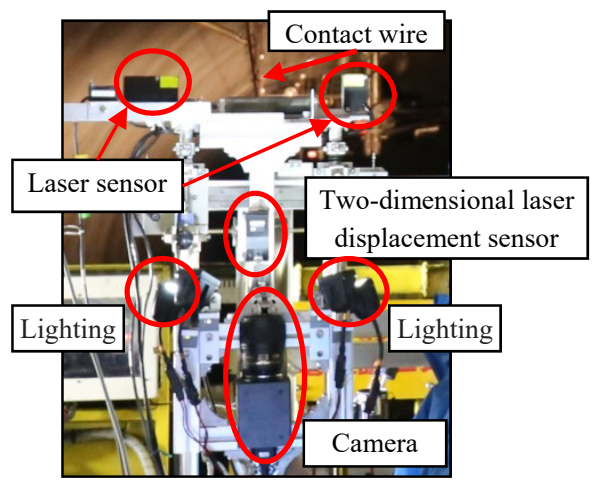

Figure 1: Measurement view by laser sensors.

Table 1: $\quad$ Specification of measuring methods.

\begin{tabular}{|c|c|c|c|}
\hline Method of measurement & Apparatus & $\begin{array}{c}\text { Sampling in the } \\
\text { direction parallel } \\
\text { with the rail [mm] }\end{array}$ & $\begin{array}{c}\text { Precision } \\
{[\mathrm{mm}]}\end{array}$ \\
\hline Static measurement & Laser scan type & 20 & 0.10 \\
\hline Electrical inspection car & $\begin{array}{c}\text { Transmission } \\
\text { laser sensor }\end{array}$ & $50(275[\mathrm{~km} / \mathrm{h}])$ & 0.10 \\
\hline
\end{tabular}


Table 2: $\quad$ Measured contact wire sections.

\begin{tabular}{|c|c|c|c|}
\hline $\begin{array}{l}\text { Section } \\
\text { no. }\end{array}$ & $\begin{array}{l}\text { Overhead contact } \\
\text { lines structure }\end{array}$ & Tunnel & Contact wire \\
\hline 1 & \multirow{2}{*}{$\begin{array}{c}\text { high-speed } \\
\text { simple catenary }\end{array}$} & presence & \multirow{3}{*}{$\begin{array}{l}\text { grooved precipitation-hardened copper } \\
\text { alloy contact wire }\left(110\left[\mathrm{~mm}^{2}\right]\right)\end{array}$} \\
\hline 2 & & \multirow{3}{*}{ absence } & \\
\hline 3 & \multirow{2}{*}{$\begin{array}{l}\text { high-speed heavy } \\
\text { compound catenary }\end{array}$} & & \\
\hline 4 & & & $\begin{array}{l}\text { grooved bronze contact wire (170 } \\
\left.\left[\mathrm{mm}^{2}\right]\right)\end{array}$ \\
\hline
\end{tabular}

\subsection{The static measurement}

In this measurement, the residual diameters and the deviation of the contact wire were measured by the measuring instrument equipped with laser sensors which can measure these values continuously in a static state. The measurement accuracy of the residual diameter by the proposed method is the same as that by the electrical inspection car. However, the laser sensors can directly measure the residual diameters from the side of the contact wire in case of this measurement. On the other hand, the width of the contact wire bottom surface is detected by the laser beam reflected by the surface of the contact wire in the electrical inspection car measurement. Therefore, the values of static measurements by the laser sensors are defined as the true values. The difference between the residual diameter of the static measurement and that by the electrical inspection car is defined as the error in this paper.

Resolutions of the two-dimensional laser displacement measuring instrument applied at the same time were $0.1 \mathrm{~mm}$ in the direction perpendicular to and $0.2 \mathrm{~mm}$ in the direction parallel with the rail. The resolution of the line camera was about $0.09 \mathrm{~mm} / \mathrm{pix}$ in the direction perpendicular to the rail.

\subsection{Measurement by the electrical inspection car}

The recording of the measured values by the electrical inspection car was carried out at the same time as the static measurement.

\section{Result of the measurement}

\subsection{Representation of the result}

The measurement results are presented in such a way that the train running direction is the standard direction. As for the contact wire deviation, with our back against the starting point, the left side and the right side have been denoted as negative and positive respectively. As for the wear surface image and the twodimensional cross-sectional shape, with our back against the starting point, the left side and the right side have been denoted "left" and "right" respectively. 


\subsection{Section no. 1}

Fig. 2(a) shows the residual diameter and the contact wire deviation measured by the laser sensors in the static state. Fig. 2(b) shows the residual diameter measured by the electric inspection car. Fig. 3 shows the wear surface image by the line camera and the two-dimensional cross-sectional shape by the two-dimensional laser displacement measuring instrument at the point indicated by the arrow in fig. 2(b). The width of the wear surface which is calculated by the residual diameter measured by the electric inspection car at the same point is shown on the wear surface image. As for the white parts in the wear surface images, they are the parts where the lighting is reflected by the gloss. The major features are shown as follows.

- There is not much unevenness on the wear surface and the arc surface at the points such as point (1) where the measurements by electric inspection car have small errors.

- The smooth glossy surface of the arc surface is not detected as the width of the wear surface by the electric inspection car.

- There is unevenness on the wear surface and the arc surface at points such as point (2) where the measurements by electric inspection car have significant errors.

- The unevenness of the arc surface is detected as the width of the wear surface by the electric inspection car.

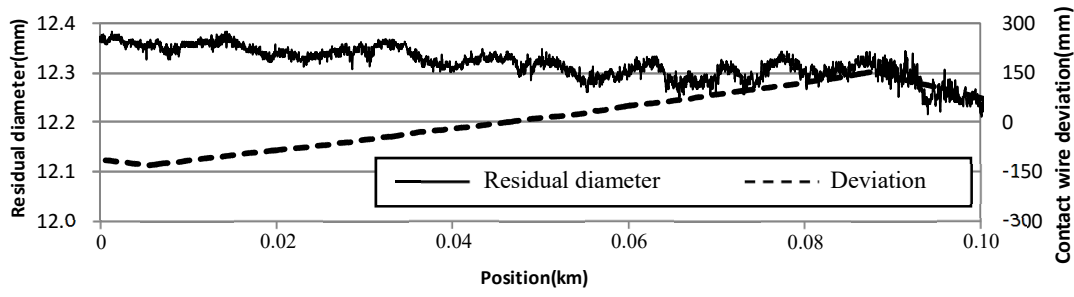

(a) Measured by laser sensors

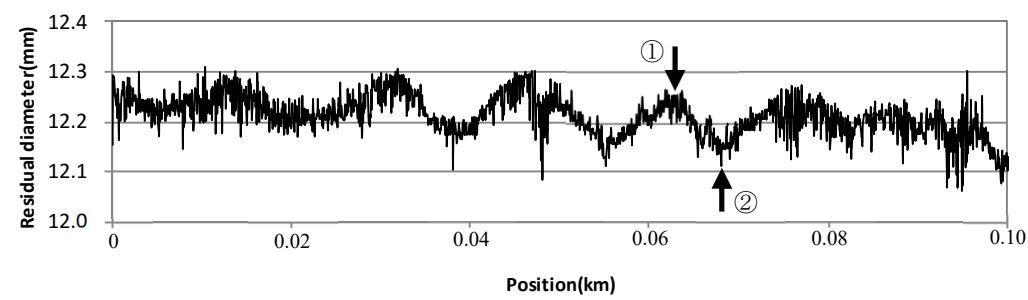

(b) Measured by electric inspection car

Figure 2: $\quad$ Residual diameter and deviation of contact wire (section no. 1). 


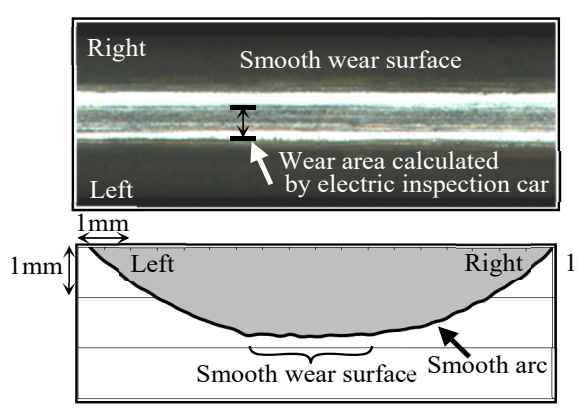

(a) Point (1)

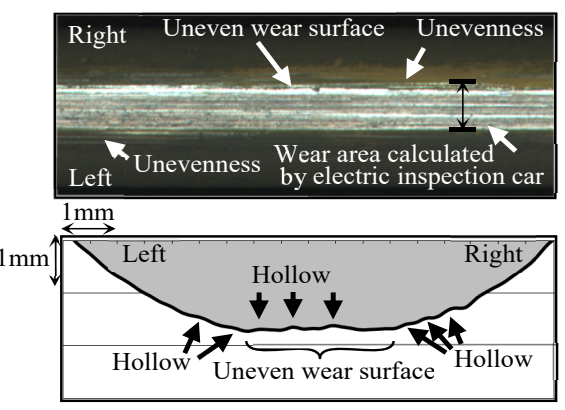

(b) Point (2)

Figure 3: Wear surface and two-dimensional cross-section of contact wire (section no. 1).

\subsection{Section no. 2}

Fig. 4(a) shows the residual diameter and the contact wire deviation measured by the laser sensors in the static state. Fig. 4(b) shows the residual diameter measured by the electric inspection car. Fig. 5 shows the wear surface image by the line camera and the two-dimensional cross-sectional shape by the two-dimensional laser displacement measuring instrument at the point indicated by the arrow in fig. 4(b). There are the following features.

- If there are edges of the wear surface at points such as point (3), the error is less likely to occur even if the arc surface is not so smooth.

- At points such as point (4) where there is unevenness on the wear surface, the error is likely to occur in the same way as in the tunnel section shown in section 3.2 .

\subsection{Section no. 3}

Figs 6 and 7 show the results of the measurements.

- At points such as point (5) where there are big errors, there is unevenness on the wear surface in the same way as that shown in sections 3.2 and 3.3, and the width of the wear surface is detected by the electric inspection car. 


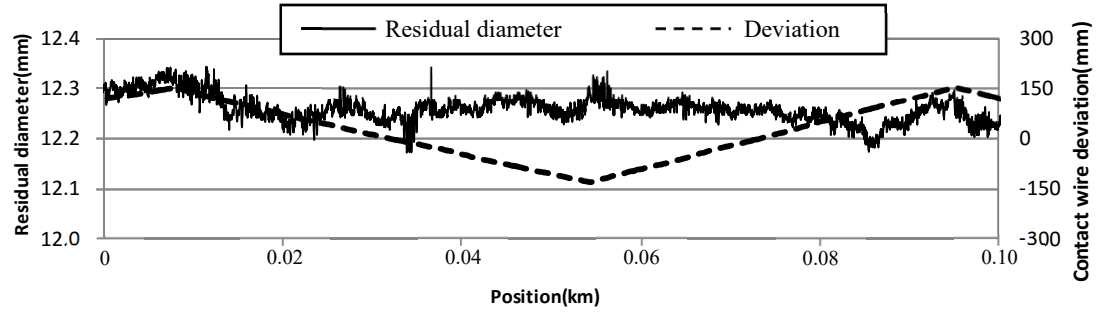

(a) Measured by laser sensors

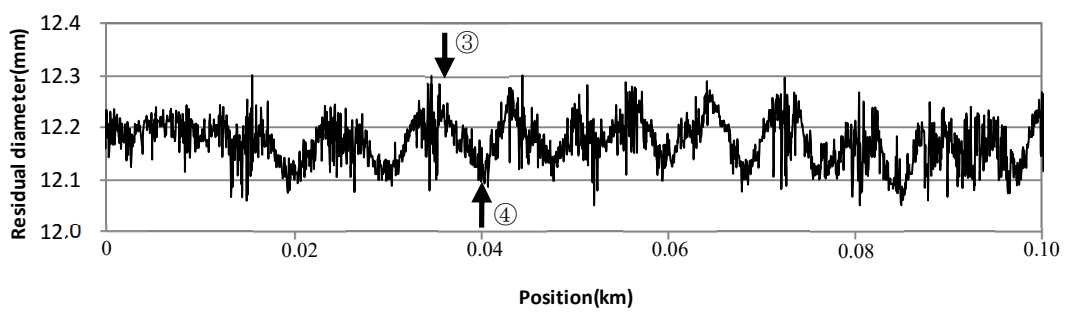

(b) Measured by electric inspection car

Figure 4: Residual diameter and deviation of contact wire (section no. 2).

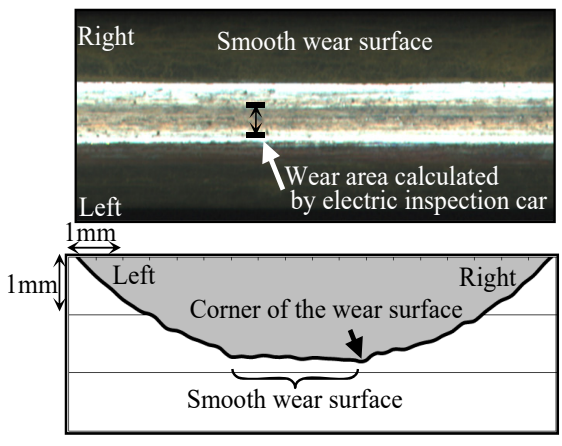

(a) Point (3)

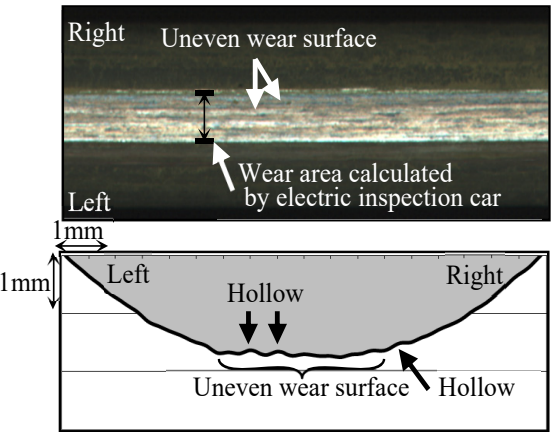

(b) Point (4)

Figure 5: Wear surface and two-dimensional cross-section of contact wire (section no. 2). 


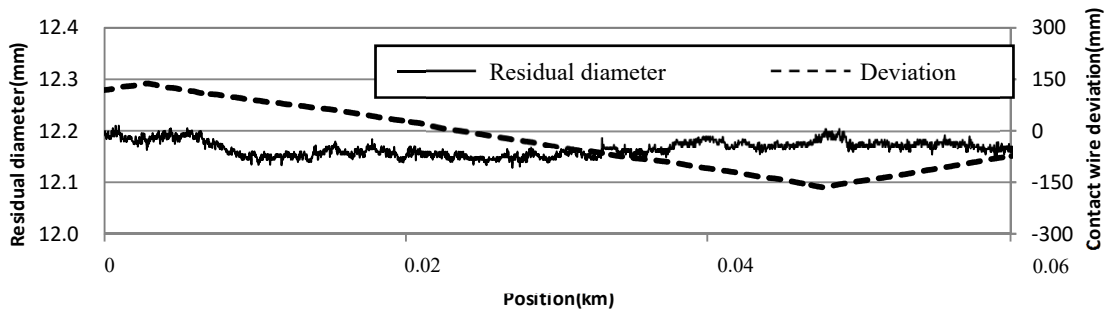

(a) Measured by laser sensors

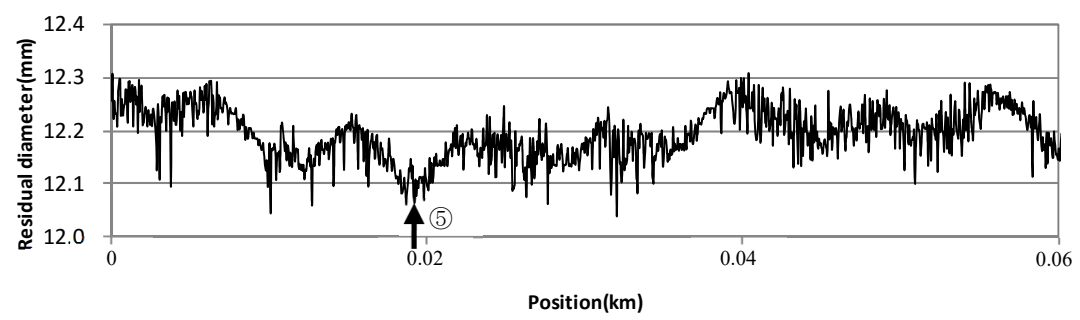

(b) Measured by electric inspection car

Figure 6: Residual diameter and deviation of contact wire (section no. 3).
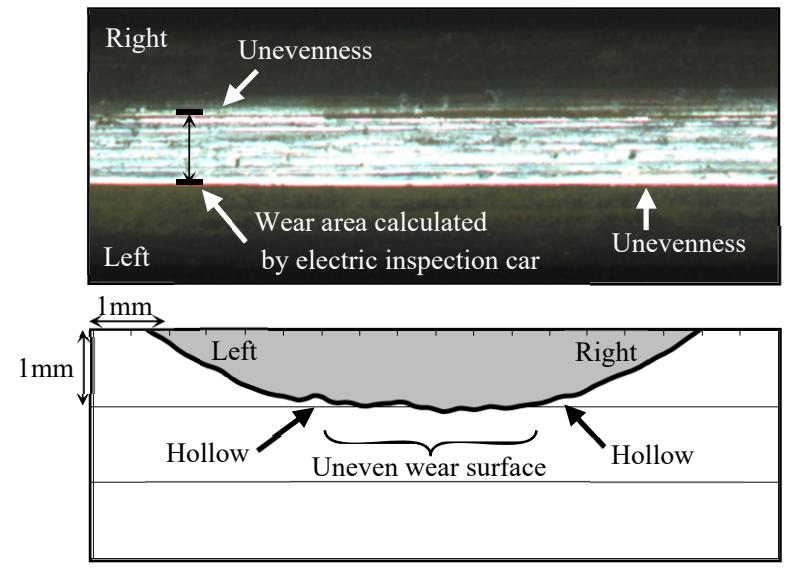

Figure 7: Wear surface and two-dimensional cross-section of contact wire (Point (5)).

\subsection{Section no. 4}

Figs 8 and 9 show the results of the measurements. There are the following features. 
- In the same way as in other sections, at the points where there is neither unevenness nor gloss on the wear surface, but there are clear edges, there are small errors.

- The gloss on the wear surface is not detected as the width of the wear surface by electric inspection car as shown at point (6).

- The edges of the wear surface are rounded and the boundary between the wear surface and the arc surface has the smooth shape at the glossy points.

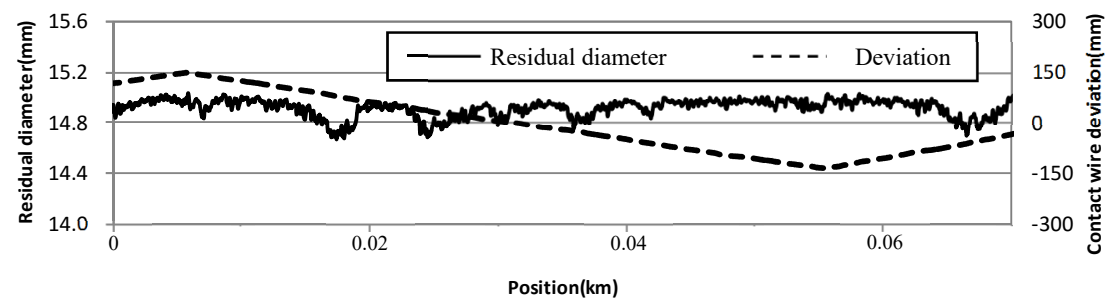

(a) Measured by laser sensors

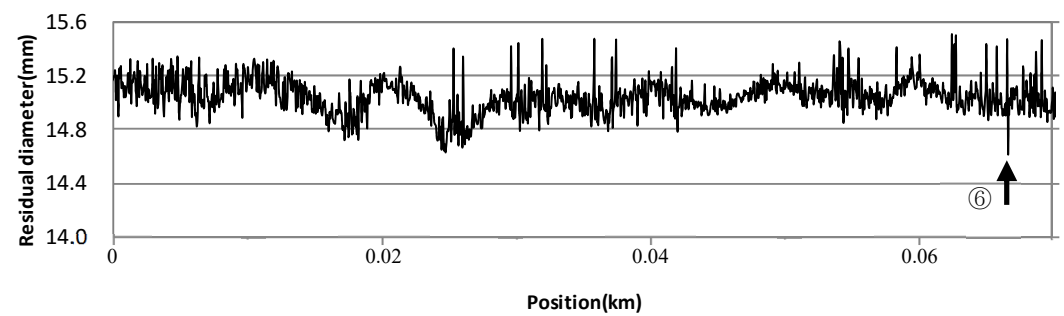

(b) Measured by electric inspection car

Figure 8: Residual diameter and deviation of contact wire (section no. 4).
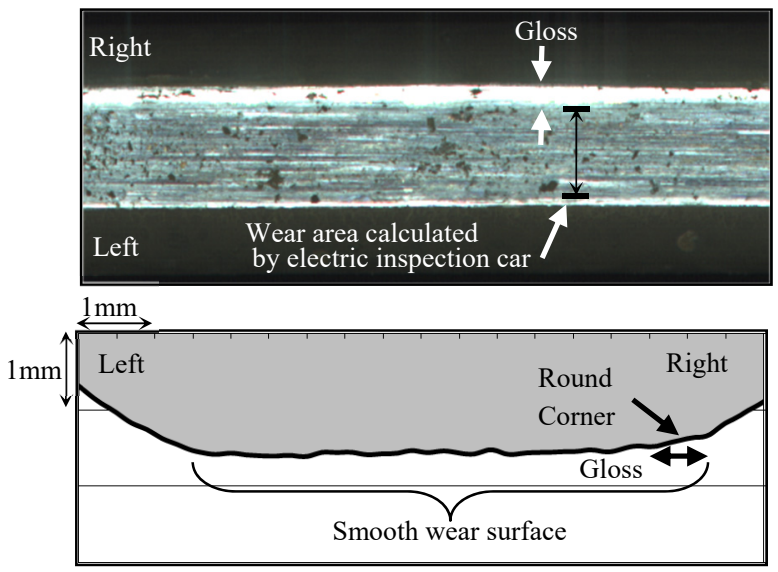

Figure 9: Wear surface and two-dimensional cross-section of contact wire (Point 6). 


\subsection{Summarization of the wear situation}

Thus, the error of the electric inspection car is estimated to be caused by the unevenness and the gloss that exist on the wear surface and on the arc surface in the vicinity which influence the reflection of the laser of the electric inspection car. In addition, it is considered that the same event may occur regardless of the existence of the tunnel, the overhead contact line structure type and the kinds of contact wire since the situations of the error occurrence points are common to the four sections.

\section{Proposal of a new measuring method}

\subsection{Proposal}

A method which can eliminate the influence by the unevenness and the gloss is proposed, since it is considered that these are the causes of the error in measurement by the electrical inspection car.

\subsection{A method utilizing the two-dimensional cross-sectional shape}

This method calculates the residual diameters by comparing the two-dimensional cross-sectional shapes which include the arc surface of the contact wire with the true circles of the new one. Fig. 10 shows the overview of this method. This method can eliminate the causes of the error since the arc surface is processed independently of the state of the wear surface. Fig. 11(c) shows the results by this method which are compared with the static measurements by the laser sensors considered to be the true values (fig. 11(a)), and the measurements by the electrical inspection car (fig. 11(b)). It is shown that the results by this method are in good agreement with the results of the static measurements by the laser sensors.

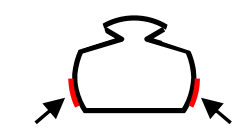

Detection of arc surface

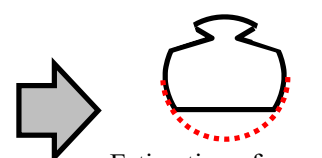

Estimation of unused shape

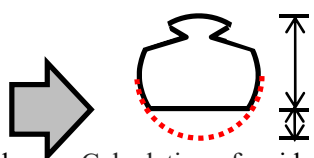

Calculation of residual diameter

Figure 10: Measured by two-dimensional cross-section.

\subsection{A method utilizing the wear surface image}

This method detects the width of wear surface that includes the glossy arc corner of the wear surface by using the strong light reflected light from there. For this purpose, the wear surface image is taken by projecting the light diagonally upward 
to the contact wire on the left and light side of the contact wire. Fig. 11(d) shows the results by this method. It is considered that this method can complement the measurements by the electric inspection car because it is possible to grasp the trend of the progression of wear state by the waveforms which are similar to those of the static measurements, although this method has a problem with respect to accuracy.

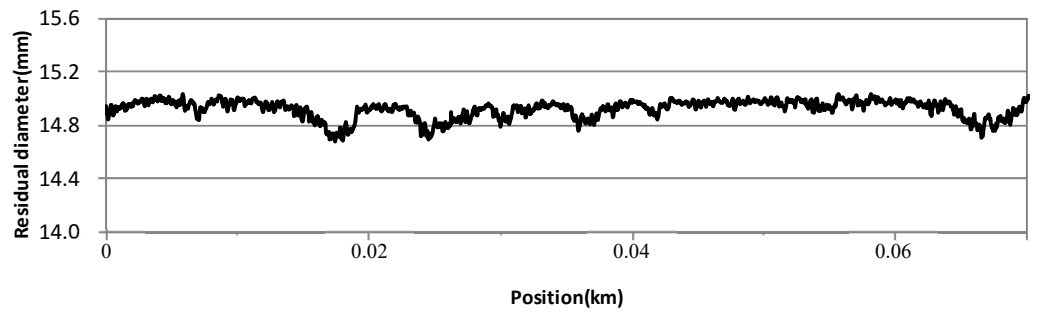

(a) Measured by laser sensors

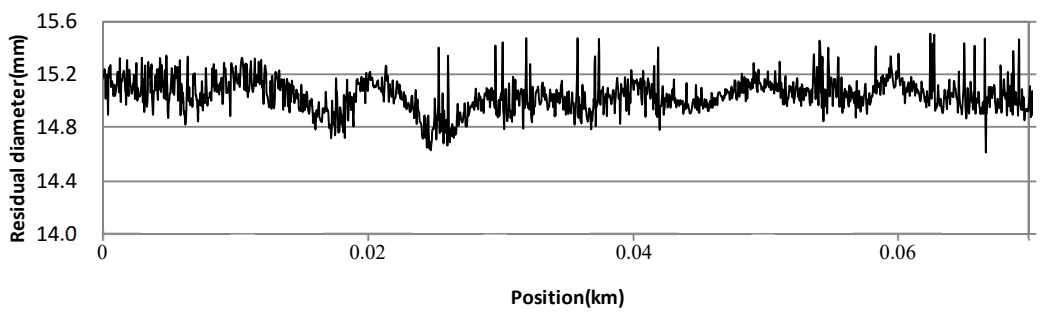

(b) Measured by electric inspection car

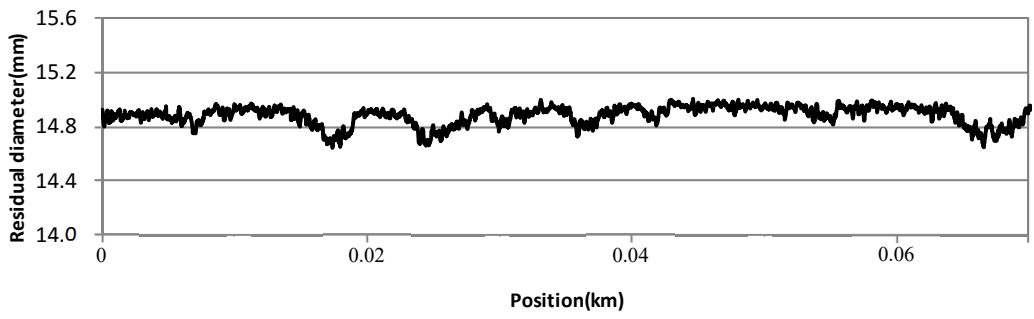

(c) Measured by two-dimensional cross-section

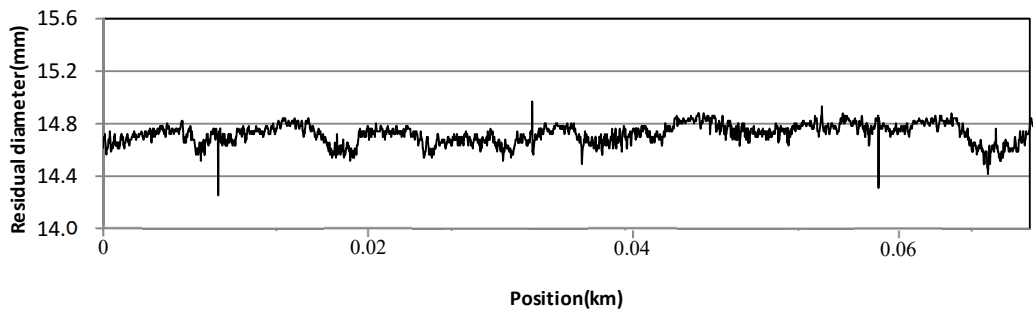

(d) Measured by image of the wear surface

Figure 11: Calculation result of proposed methods. 


\section{Conclusion}

As for the wear of the contact wire, based on the comparison between the static measurements and the measurements by the electric inspection car, it was confirmed that unevenness and gloss exist at the points where the error is large regardless of the existence of the tunnel, the types of the overhead contact lines structure, and the kinds of contact wires.

It is considered that these are the causes of the error of the measurement by the electrical inspection car. The methods utilizing the two-dimensional crosssectional shape and the wear surface image are proposed to eliminate these causes of the errors. It is confirmed that the proposed methods are complementarily valid to the measurement by the electric inspection car, by comparing the measurements by these methods with the static measurements and the measurements by the electric inspection car. However, the measurements by the proposed methods were conducted at low speed at the location near the contact wire. In introducing these methods to the electric inspection car, it is necessary to install the relevant apparatus so that these sizes and positions do not invade the rolling stock gauge, while also retaining at high speed the resolution level comparable to the one used this time.

In the future, we will continue to examine the trend and the mechanism of the unevenness and gloss on the wear surface along with the introduction of the proposed methods to the electric inspection car.

\section{References}

[1] Sato, H., et al., New Shinkansen Electric \& Track Inspection Car (East-i). Railway \& Electrical Engineering, Vol. 13, No. 10, pp. 12-16, 2002 (in Japanese).

[2] Kusumi, S., Apparatus for Measuring Wear of Contact Wire on Electric Inspection Car. RRR, Vol. 65, No. 1, pp. 34-35, 2008 (in Japanese).

[3] Harada, S., et al., Examination of Contact Wire Wear Condition in Tunnel Section on Shinkansen. Japan Industry Applications Society Conference, No. 3-56, pp. 307-308, 2008 (in Japanese).

[4] Honda, M., et al., Measurement of Cross-section of Contact Wire in Shinkansen Tunnel. Japan Industry Applications Society Conference, No. 518, pp. 195-198, 2014 (in Japanese). 